\title{
Efeitos da atividade física sobre a densidade mineral óssea de mulhe- res saudáveis na pré-menopausa
}

\section{Physical activity effects on bone mineral density of healthy women in pre-menopause}

\author{
Cristiane F. F. Silva ${ }^{1}$; Elaine S. C. Rodrigues ${ }^{1}$; Antônio J. Natalii ${ }^{2}$ Luciana M. Lima $^{3}$
}

\begin{abstract}
RESUMO
Modelo de estudo: Revisão sistemática da literatura

Objetivo: A atividade física representa um importante estímulo ao aumento da densidade mineral óssea (DMO). Como a resistência dos ossos está associada tanto a DMO, quanto a microestrutura e propriedades do material, melhorias na massa óssea são importantes na prevenção de osteoporose na idade mais avançada. Apesar de muitos estudos sobre o tema, não existe consenso sobre qual seria o melhor tipo de atividade física, intensidade e frequência para melhorias na osteogênese e promoção do ganho de DMO. O objetivo deste estudo foi investigar os tipos de atividades físicas com melhor efeito osteogênico de aumento da DMO em mulheres saudáveis na pré-menopausa.

Metodologia: Realizou-se levantamento bibliográfico de artigos científicos nas bases de dados Pubmed e Direct Science publicados nos últimos dez anos. Foram selecionados estudos controlados com exercícios de alto impacto e resistido. Utilizou-se análise sistematizada dos artigos selecionados.

Resultados: Foram incluídos 15 artigos nesta revisão, os quais foram separados em dois grupos, conforme o tipo de exercício. Foi realizada análise comparativa de metodologias utilizadas e resultados alcançados. Constatou-se maior eficiência do protocolo de exercício de alto impacto, mesmo em curto período de execução para aumento da DMO do fêmur proximal (colo femoral e região intertrocantérica). Em menor frequência, foi observado aumento da DMO na coluna lombar quando exercícios resistidos ou de impactos maiores foram usados.

Conclusões: A maioria dos artigos executou protocolo experimental em período relativamente curto (6 meses), demonstrando que programas de exercícios simples, de fácil execução, curto período de aplicação e sem uso de aparelhos específicos são suficientes para promover remodelação óssea em sítios específicos com aumento da DMO.
\end{abstract}

Palavras-chaves: Exercício. Densidade Mineral Óssea. Mulheres.

1. Mestranda do Departamento de Educação Física, Universidade Federal de Viçosa, Viçosa, Minas Gerais

2. Professor Associado, Departamento de Educação Física, Universidade Federal de Viçosa, Viçosa, Minas Gerais

3. Professora Adjunta, Departamento de Medicina e Enfermagem, Universidade Federal de Viçosa, Viçosa, Minas Gerais
Correspondência: Profa. Dra. Luciana Moreira Lima Universidade Federal de Viçosa, Departamento de Medicina e Enfermagem Av. PH Rolfs, $s / n$ - Centro CEP 36570-000 Viçosa, MG, Brasil E-mail: luciana.lima@ ufv.br

Artigo recebido em 12/03/2013 Aprovado para publicação em 20/02/2014 


\section{Introdução}

A literatura demonstra que uma das formas eficientes de aumentar a densidade, a resistência e a massa óssea de maneira não farmacológica é a prática de atividade física. ${ }^{1}$ Essa pode afetar positivamente os níveis de hormônios que são essenciais para o metabolismo do tecido ósseo, diminuindo os riscos de fraturas ao longo da vida., ${ }^{1,2}$

O tecido ósseo é altamente dinâmico, formado e remodelado constantemente, sendo importante a manutenção de sua homeostasia. A ruptura desse equilíbrio pode levar a importante problema enfrentado pela saúde pública - a osteoporose $\mathrm{e}^{2-5}$ - que acomete principalmente mulheres na menopausa. ${ }^{2}$ Caracterizam-se por diminuição acentuada da DMO e alteração das propriedades, arquitetura e resitência dos ossos, aumentando a predisposição à fratura. ${ }^{6}$ No metabolismo ósseo, o estado hormonal, em especial nas mulheres, é de grande relevância, sendo o ciclo menstrual e sua regularidade, essenciais para homeostasia óssea. ${ }^{7,8}$ Receptores para estrógeno existem nos osteoblastos ${ }^{7,9}$ e parecem aumentar a capacidade adaptativa do osso às forças de tensão as quais é submetido ${ }^{10}$ por meio de maior produção de matriz óssea.

Vários estudos têm demonstrado que a atividade física produz efeito osteogênico importante, com auxílio no desenvolvimento ósseo, aumento da DMO, ${ }^{2,5,11,12,13}$ além de melhorar a capacidade neuromuscular, diminuindo a frequência de quedas. ${ }^{12,13} \mathrm{E}$ estratégia utilizada não só em mulheres na pré-menopausa, ${ }^{12}$ como também na adolescência e juventude, auxiliando, juntamente com outros fatores, no aumento do pico de massa óssea, prevenindo fraturas na idade mais avançada. ${ }^{2,3,12,14}$ No estudo de KEMPER e col. (2002)15 foi demonstrado via pontuações do questionário de atividade física (PAQ) em adolescentes, que adaptações ósseas ocorrem continuamente, com ajuste da massa e arquitetura óssea em resposta às solicitações mecânicas, ${ }^{12,15,16}$ melhorando o pico de massa óssea em mulheres na pré-menopausa. Essa resposta observa a lei de Wolff de que a DMO será maior de acordo com a magnitude e direção da força aplicada sobre dada região do osso. ${ }^{17}$

O exercício de alto impacto tem sido apontado como o mais efetivo em relação à melhora da DMO do colo do fêmur, ${ }^{2,4,18,19}$ conforme estudos utilizando breves e regulares exercícios de saltos que indicaram ganho de DMO no quadril de mulheres na pré-menopausa. ${ }^{11,13}$ Além disso, pesquisas comparando atletas de diferentes esportes, apontam que praticantes de esportes de alto impacto têm maior DMO os demais. ${ }^{3,13}$

Apesar das diversas pesquisas sobre os efeitos dos exercícios na modelação e remodelação óssea, o tipo, frequência, intensidade e duração dos mesmos ainda não estão totalmente consolidados pela literatura. ${ }^{8,13,15}$ Além disso, poucos são os estudos prospectivos controlados, randomizados em mulheres saudáveis na pré-menopausa. ${ }^{3,18}$

Algumas dificuldades foram observadas neste estudo, como presença de resultados contraditórios, possivelmente devido à variedade de metodologias, protocolos de exercícios e pouca aderência aos procedimentos. Em função disso, pesquisas buscam comprovar eficácia de programas fáceis e rápidos, aplicados no trabalho ou tempo livre. ${ }^{5}$

O objetivo dessa revisão é auxiliar na escolha do protocolo de exercícios que melhore a DMO de mulheres na pré-menopausa como forma de prevenção e promoção da saúde.

\section{Métodos}

\section{Estratégia de busca}

Foi realizada em agosto de 2012 pesquisa nas bases de dados on-line Pubmed e ScienceDirect referente ao período dos últimos dez anos até a data atual (2002 a 2012), nas línguas portuguesa e inglesa. Utilizou-se na pesquisa pelo site ScienceDirect os indexadores: "bone" e "premenopausal" em "(title/ abstrat/ keywords)", sendo encontrados um total de 78 artigos. Foram excluídos os artigos de revistas e jornais que tratavam de doenças (câncer, alterações reumatológicas, pulmonares, gastrointestinais, psiquiátricas, ósseas e musculares), procedimentos após intervenção cirúrgica (bariátrica, ortopédica e transplante) e uso de medicamentos ou reposição hormonal, sendo selecionados 58 potenciais artigos para a revisão.

Paralelamente, a partir de levantamento bibliográfico no site Pubmed, utilizando a pesquisa avançada com os seguintes indexadores: em "Title" - "bone"; em "Title/abstract" - "premenopausal", "women", "exercise"; e em "data publication" - de janeiro de 2002 a julho de 2012, foram selecionados 100 artigos, dos quais foram excluídos os relacionados a problemas de saúde (câncer, fibromialgia, sobrepeso, amenorréia, anorexia, sarcopenia, osteopenia, osteoporose e epilepsia), além dos referentes ao tabagismo, períodos menopausal e pós-menopausal, assim como aqueles 
que não mencionavam atividade física utilizada em seus protocolos experimentais. Foram selecionados na Pubmed para este estudo 48 potenciais artigos.

Após a busca dos artigos e submissão aos critérios de exclusão, foram selecionados 15 artigos para comporem o estudo, sendo subdivididos segundo os tipos de protocolos de exercícios: de alto impacto, resistidos e/ou aeróbicos sem alto impacto, de acordo com o fluxograma (figura 1).

\section{Critérios de inclusão e exclusão}

Foram incluídos nesta pesquisa apenas estudos que apresentaram experimentos com protocolos de atividades físicas com grupo controle em mulheres saudáveis na pré-menopausa e relataram os efeitos do exercício na DMO. Utilizaram-se os seguintes critérios de exclusão: mulheres na menopausa ou pósmenopausa, pesquisas que não mencionavam a atividade física, doenças associadas, uso de medicamentos ou hormônios e ausência de grupo controle. Também foram excluídos artigos em que as atividades físicas eram relatadas somente em questionários e os estudos de revisão de literatura.

\section{Análise}

Os artigos foram divididos em dois grupos de acordo com o protocolo de treinamento utilizado: exercícios com alto impacto (Tabela 1) e exercícios resistidos e/ou aeróbicos sem alto impacto (Tabela 2). As características de cada grupo de exercícios e os efeitos sobre a DMO de mulheres na pré-menopausa foram comparadas e analisadas.

\section{Resultados}

Dos 175 artigos selecionados inicialmente, 100 eram da Pubmed e 78 da Direct Science, sendo que todos passaram pelos mesmos critérios de inclusão: mulheres na pré-menopausa e efeito da atividade física na DMO, restando 106 artigos. Desse conjunto de artigos foram selecionados 15 (figura 1), a partir da submissão ao seguinte critério de exclusão: estudos com grupo controle cujo protocolo experimental de atividade física era descrito detalhadamente constando informações necessárias a análise, como frequência, intensidade e duração.

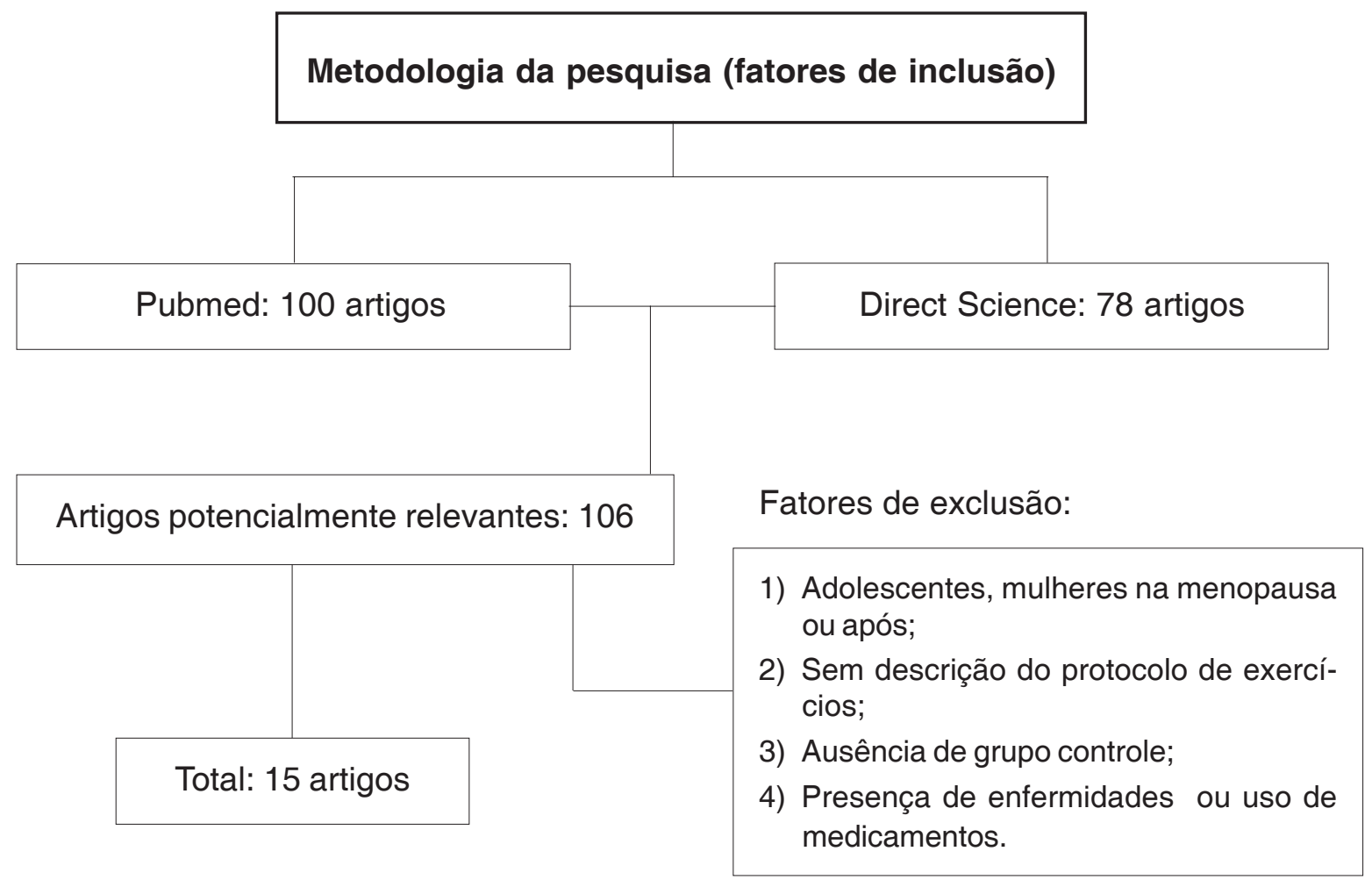

Figura 1: Fluxograma com a metodologia utilizada no estudo. 
Os artigos incluídos nessa revisão foram divididos em grupos de acordo com o tipo de atividade: protocolos de alto impacto de exercícios (10 artigos) e protocolos de exercícios resistidos e/ou aeróbicos sem alto impacto (5 artigos). Foi realizada análise sistematizada das alterações em relação à DMO e às características das atividades físicas de cada grupo de protocolos, comparando-as em seguida. Alguns artigos adicionais foram usados como suporte teórico.

\section{Discussão}

Um dos problemas mais comuns associados à osteoporose é a fratura por fragilidade óssea, comuns no quadril e vértebras da coluna, sendo a coluna mais sensível às mudanças de circulação dos hormônios sexuais pela alta quantidade de osso trabecular. ${ }^{13,17}$ Em relação às mudanças ocorridas na DMO, observou-se comportamento diferente de locais específicos do corpo em relação ao tipo de protocolo de exercício utilizado, como também, mecânica e local de aplicação da carga. ${ }^{8}$ Isso poderia facilitar uma prescrição mais direcionada a locais específicos de suscetibilidade a fraturas por fragilidade, por exemplo, o colo femoral onde há afinamento do osso cortical. ${ }^{13}$ Em adição, notou-se relação entre a frequência de treinamento e a extensão das mudanças ósseas, sendo importante a regularidade de treinamento ${ }^{13,17}$ e o tempo de treinamento, relativamente curto, necessário para que essas mudanças ocorram. ${ }^{17}$ Mas, os aumentos de massa óssea em mulheres na pré-menopausa são em torno de 2 - 3\% na DMO, e essa pequena porcentagem parece ocorrer devido à lenta mudança. ${ }^{14}$ Observa-se também que efeitos anabólicos podem estar relacionados à intensidade dos estímulos mecânicos que devem ultrapassar o limite mínimo de intensidade de carga e serem aplicadas de forma menos usual para sua ocorrência. ${ }^{15}$

Nos estudos que utilizaram protocolos de exercício com alto impacto (Tabela 1), observou-se melhora da DMO do quadril, principalmente no colo femoral, mas poucos relataram aumento da DMO da coluna lombar. Esse fato pode estar relacionado com a duração do protocolo (variou de 6 a 12 meses), sendo talvez curto para promover alterações na DMO de outros sítios ósseos específicos. ${ }^{13}$ Deve-se esclarecer que o ciclo completo de ativação, reabsorção e formação dura em torno de 3 a 4 meses e que são necessários vários ciclos completos para mudanças que sejam detectadas ao exame convencional (Dual-energy Xray Absorptiometry - DXA). Alguns estudos sugerem que essa diferença ocorra em razão da maior força tensional de flexão e compressão sobre o quadril nos exercícios com peso e força de reação do solo, ${ }^{10}$ tanto na impulsão quanto na aterrissagem ${ }^{4}$ e sua dissipação nessa articulação, amenizando sua intensidade ao alcançar a coluna lombar. Outros estudos citam que o exercício de alto impacto também ocasiona aumento da DMO da primeira vértebra lombar (L1). Porém, ao medir a DMO da coluna lombar muitos protocolos descartam a primeira vértebra lombar fazendo medidas somente de L2-L4. Outros relatam que o aumento de DMO em L1 deve-se à sua função mecânica, por se tratar de vértebra de transição entre a região torácica e lombar, e, assim, estar sob o efeito de várias direções de força e tensão.

Assim, exercícios aeróbicos que envolvam forças de reação do solo apresentam maior eficácia na readaptação óssea com aumento da DMO em sítios ósseos que recebem maior força tensional, por exemplo, colo do fêmur e regiões intertrocantéricas. E o efeito sobre a massa óssea no adulto está mais relacionado com a intensidade de tensão mecânica proporcionada pelo sistema muscular e /ou forças de reação do solo do que propriamente o gasto energético metabólico, ${ }^{15}$ sendo que nos adolescentes isso está mais relacionado a atividades com maior gasto energético. $^{16}$

Outros estudos têm demonstrado a eficácia de programas breves de saltos verticais em mulheres saudáveis na pré-menopausa. ${ }^{4,13}$ Por exemplo, o estudo de Niu e col. (2010), em que 91 mulheres prémenopáusicas randomizadas e dois grupos tratados, um com alongamento e o outro com alongamento e saltos verticais, apresentaram melhora nas medidas de DMO pelo DXA no colo femoral e na coluna lombar e que esse aumento se deveu, provavelmente, as tensões dinâmicas geradas no tecido. ${ }^{4}$ Outra característica presente nos protocolos é a progressão do programa de exercícios com aumento da força, importante para continua adaptação, ${ }^{13}$ e para isso algumas pesquisas utilizam o acelerômetro para controle dessa progressão. ${ }^{4}$ Esse dispositivo de medida do movimento tem sido bem aceito pela sua praticidade e razoável reprodutibilidade. ${ }^{12}$ As intensidades dessas acelerações durante os exercícios têm demonstrado correlação com as cargas de impacto, ou seja, forças de reação do solo. E assim, supõe-se que altos picos de aceleração estão associados a altos picos de tensão. ${ }^{12,14}$

A questão do efeito da intensidade da força de reação é importante, sabe-se que a resposta osteogênica ocorre quando a tensão excede o limite mínimo, ${ }^{10}$ 


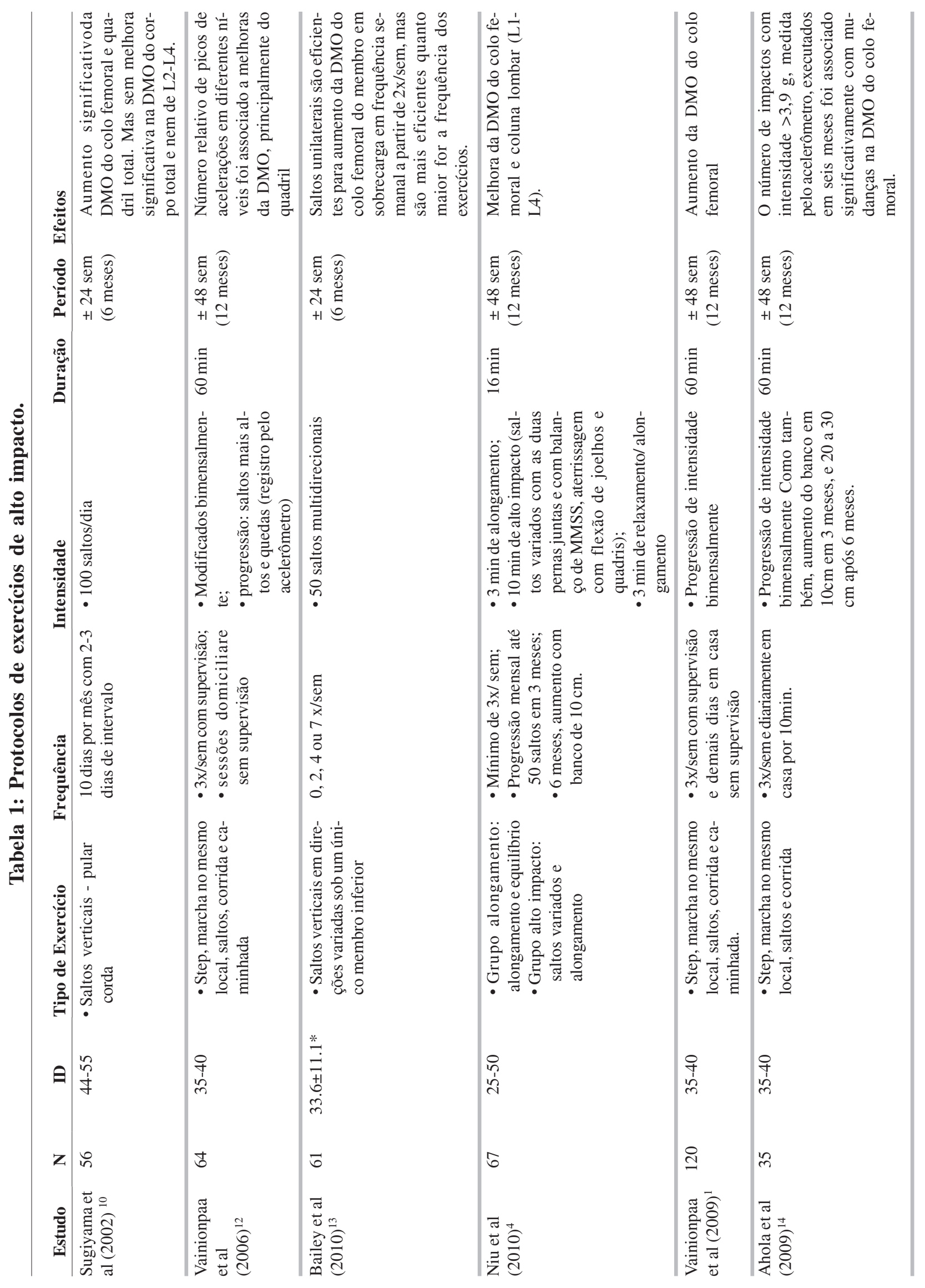



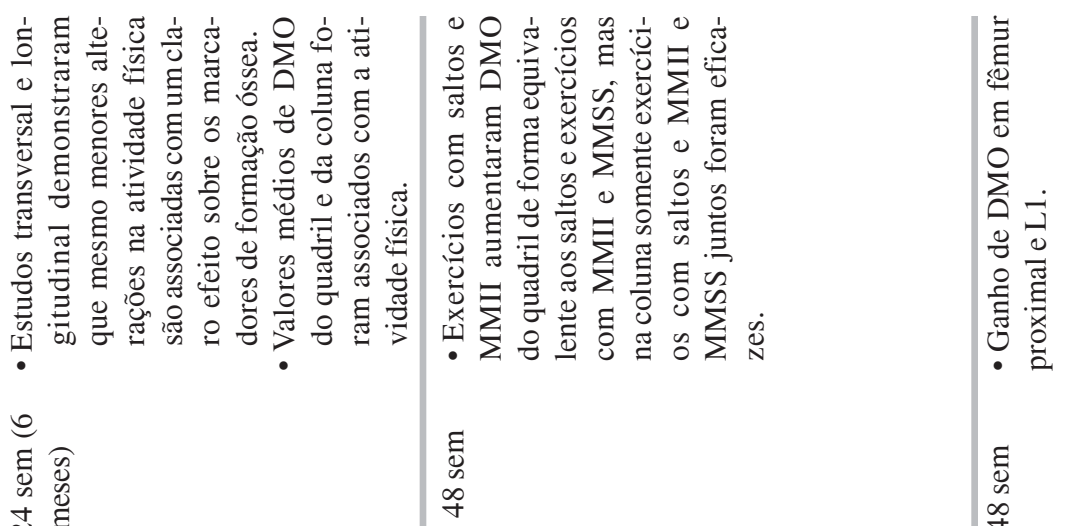

e

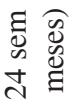

$\stackrel{\infty}{+}$

$+1$

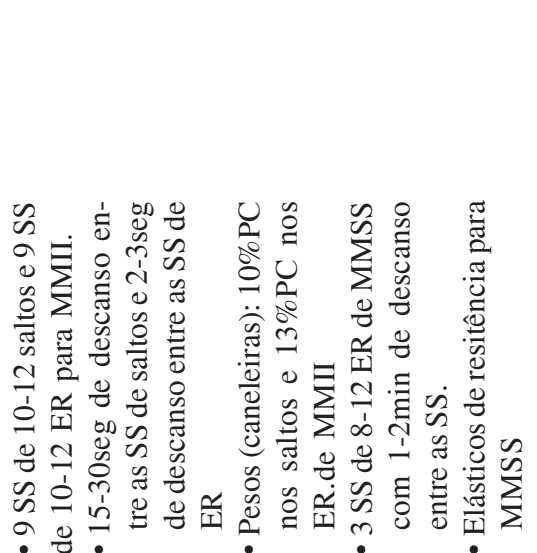

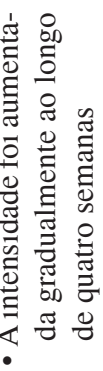

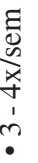

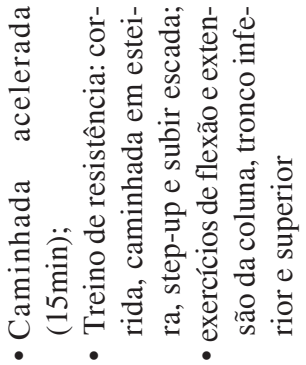

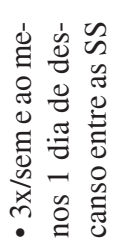

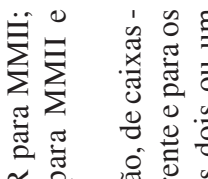

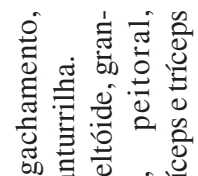

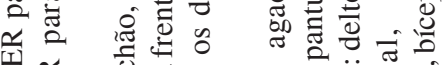

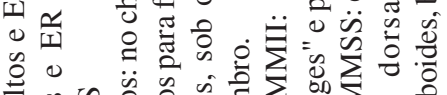

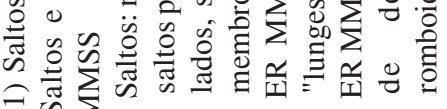

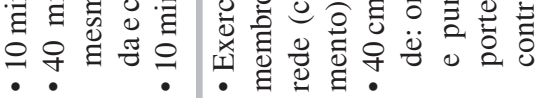

字

ले

त

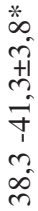

$\stackrel{4}{(-10}$

in

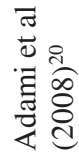

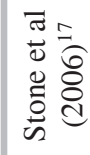
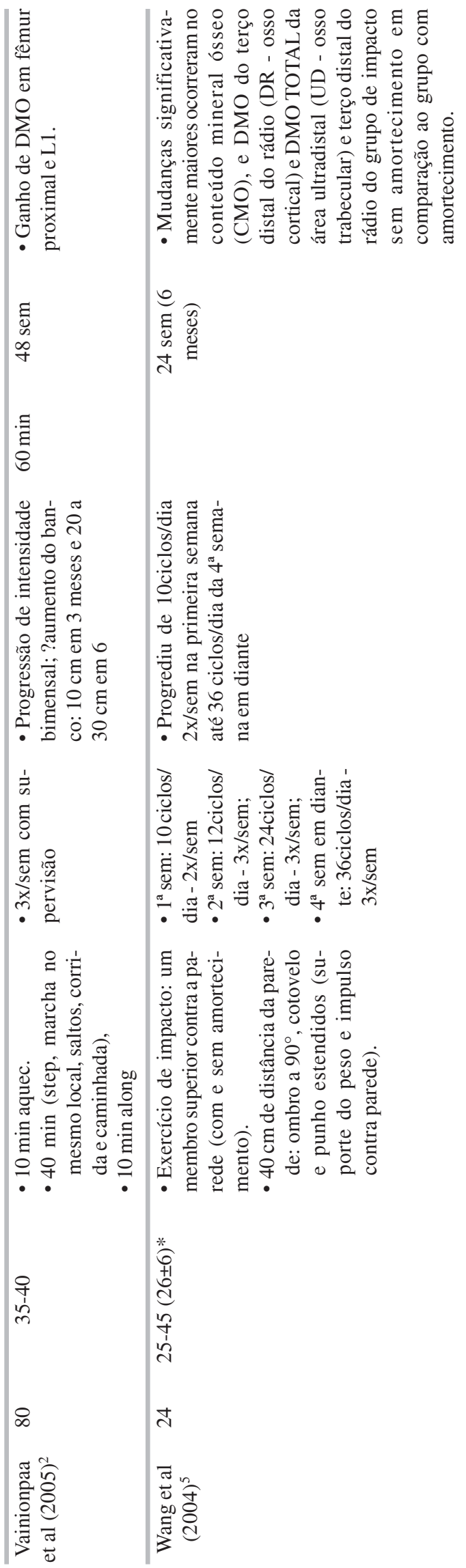


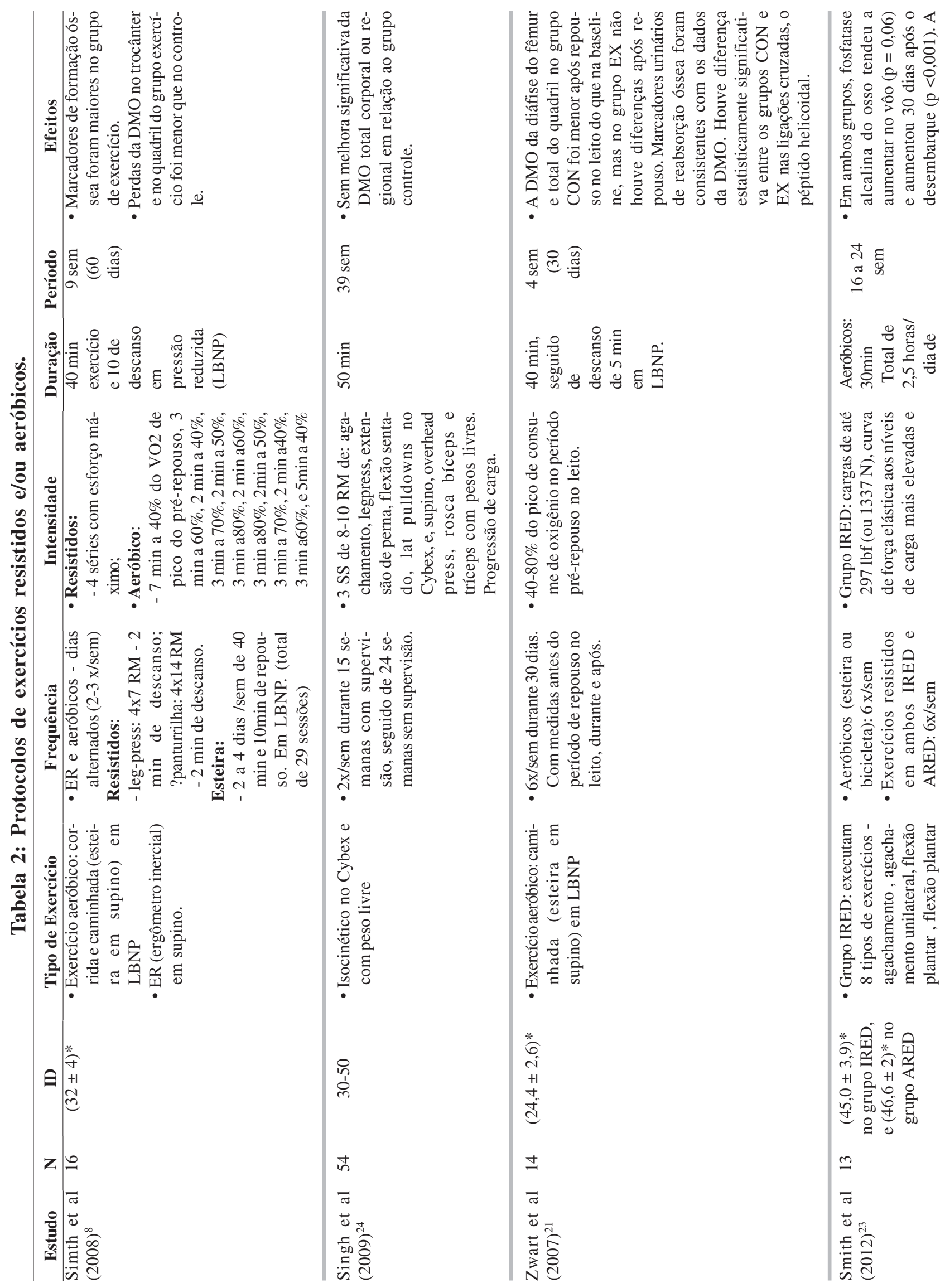




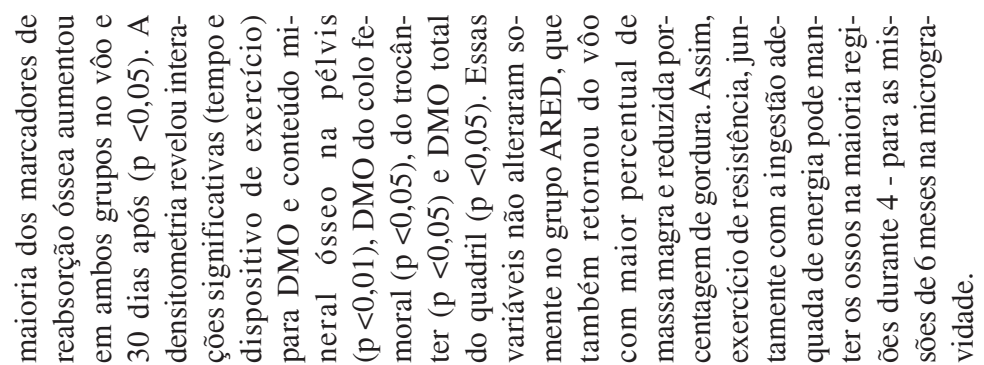

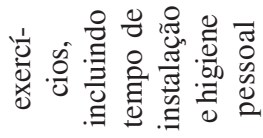

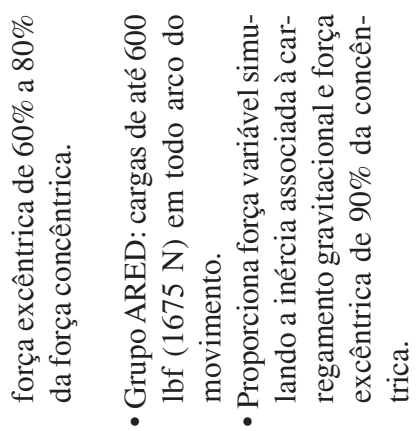

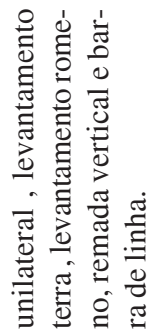

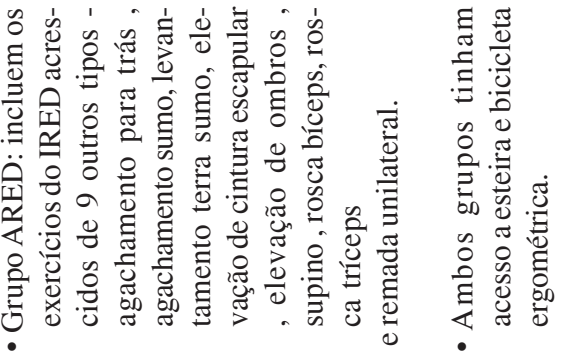

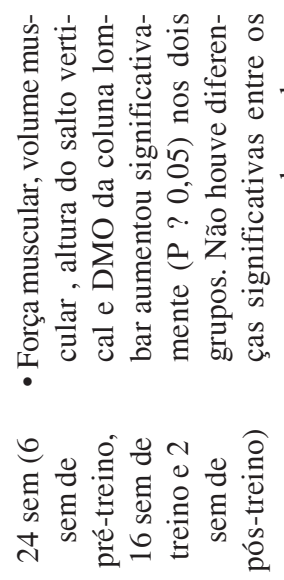

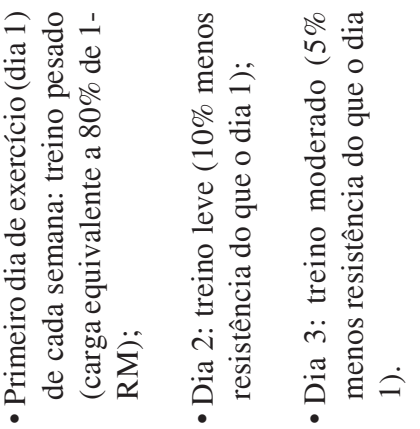

$\frac{\pi}{\sqrt[0]{n}}$

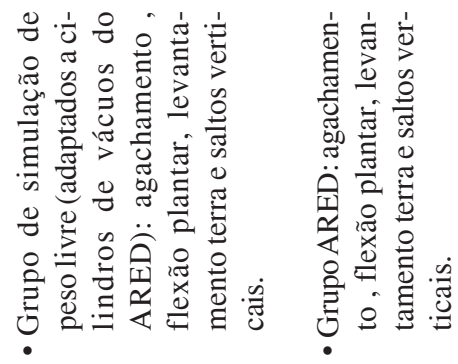

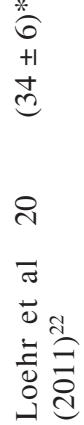

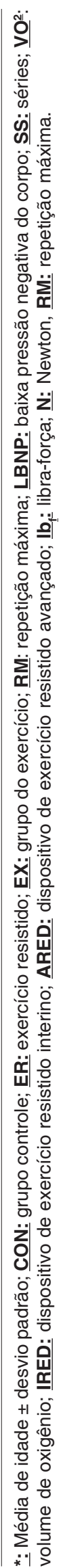


assim como também a frequência de carga. No estudo de BAILEY e col (2010), indivíduos que realizaram maior frequência dos exercícios por semana e com maior intensidade obtiveram maior aumento da DMO no quadril e também em intensidades ainda mais elevadas na coluna lombar. ${ }^{13}$ No estudo de WANG e col (2004), ${ }^{5}$ utilizando novo protocolo de impacto com um único membro superior contra a parede, com carga associada à distância, número de ciclos e frequência do exercício, a análise de regressão linear previu que forças de impacto de $345 \mathrm{~N}$ são necessárias para promover aumento na DMO do rádio distal. E ainda, que aumentos de $0,02 \mathrm{~g} / \mathrm{cm}^{2}$ na DMO resultam de cada aumento de $100 \mathrm{~N}$ por segundo no impulso. Porém, essas mudanças ocorreram no terço distal do rádio, mas não na extremidade mais distal. Estes autores relacionaram essas diferenças no comportamento às diferentes constituições ósseas em cada local, sendo o terço distal composto por osso cortical e a extremidade mais distal constituído de osso trabecular. Essas diferentes estruturas ósseas possuem diferentes propriedades mecânicas, onde o osso cortical é mais rígido e mais duro do que o trabecular, contudo esse último apresenta maior capacidade de estocar energia, isto é, mais flexível. Essas propriedades distintas são devido às diferenças na porosidade, nível de mineralização e orientação das fibras colágenas e respondem de forma diferente aos exercícios. Também, exercícios de salto em uma única perna ocasionam maior carga biomecânica de aplicação de força no membro inferior, em torno de 2,5 vezes o peso do corpo na baseline pré-intervenção e aumenta para aproximadamente 2 , 8 vezes o peso do corpo após intervenção com o exercício, correspondendo a aumento de $15 \% .{ }^{13}$ Portanto, parece ter maior efeito de proteção e aumento da DMO em regiões específicas de estresse causadas pelos saltos unilaterais do que com ambos os membros inferiores juntos, 8 porém esse tipo de intervenção deve ser utilizado com cautela pelo maior risco de lesões.

Um dos estudos selecionados ${ }^{17}$ utilizou um protocolo combinando exercício de alto impacto com exercício resistido. Eles observaram que a DMO do quadril aumentava quando associada aos exercícios resistidos de membros inferiores (MMII) combinados com saltos e resistidos de MMII e membros superiores (MMSS) também combinados com saltos. Estes resultados são semelhantes aos protocolos que utilizaram somente saltos, pois nessas situações houve aumento significativo da DMO do quadril. Porém, observou-se que a DMO da coluna também aumentou quando se associou exercícios de força para MMSS.
Uma possível explicação para esse aumento de DMO na coluna pode ser o fato de maior ativação dos músculos posturais da coluna, uma vez que este treino de força foi executado com elásticos de resistência que solicitam força de estabilização do tronco. Entretanto, como não houve grupo que executasse somente protocolo de resistidos com MMSS, essa suposição deve ser averiguada com outros estudos.

Com relação aos protocolos de exercício resistidos e/ ou aeróbico sem alto impacto, estudos que envolvem simulações de ambientes anti-gravitacionais, como vôos espaciais, têm sido desenvolvidos com auxílio de exercícios aeróbicos e resistidos separadamente, e em combinação, no intuito de avaliar a melhor forma de prevenção de perda óssea pelo desuso e pela falta de impacto ou forças de reação do solo.

A literatura tem demonstrado o benefício da atividade física nessas situações com resultados de aumento dos biomarcadores de formação óssea, apesar dos marcadores de reabsorção também continuarem elevados. ${ }^{8}$ No estudo de Smith e colaboradores ${ }^{8}$ alegou-se menor nível de capacidade e menor velocidade na execução de exercícios na esteira dos indivíduos do sexo feminino, tendo assim, menor carga e gradientes de pressão hidrostática dentro dos vasos sanguíneos e colunas de outros fluidos no corpo. Porém, o equilíbrio homeostático do metabolismo ósseo foi ainda favorável. O estudo demostrou ainda que a combinação de exercícios aeróbicos com resistidos promoveu a formação óssea, porém em menor porcentagem que nos exercícios de impacto. Foi relatado pelos autores que exercício resistido também acarreta aumento da formação óssea enquanto produz pouco efeito sobre a reabsorção óssea em pacientes no leito. Embora os exercícios resistidos possam causar estímulo independente de carga óssea - sendo essa também possivelmente mais tensional - e preservar a força muscular durante a falta de carga de impacto, esta modalidade de exercício pode preservar a capacidade de geração de força pelo músculo para proteção óssea. ${ }^{8}$ Nesse estudo em que foi simulado ambiente antigravitacional pode-se, mais uma vez, evidenciar o peso como fator importante na manutenção da resistência óssea. Como as forças de reação do solo nos ambientes de gravidade normal são iguais ao valor do peso do indivíduo nas posturas estáticas de pé e podendo ser aumentadas em multiplas vezes dependendo da atividade executada, como já mencionado anteriormente, isso não ocorre em ambientes sem gravidade e o estímulo à formação óssea é diminuido, enquanto a reabsorção continua ocorrendo. Corroborando com 
está questão ZWART e col (2007) ${ }^{21}$ observaram que utilizando o mesmo protocolo com pares de gêmeos do sexo feminino os resultados foram menos significativos que num estudo prévio com homens e que isso provavelmente seria devido à maior massa corporal total dos indivíduos do sexo masculino, maior aptidão física para alcançarem maiores velocidades na esteira e gerarem, dessa forma, maiores picos de força de reação do solo. Mantendo essa linha de pesquisas em ambientes gravitacionais e após verificação da pouca influência de exercícios aeróbicos e resistidos como contramedida eficaz de perda óssea nesses ambientes similares a vôos espaciais, Loehr et al $(2011)^{22}$ e SMITH et al (2012) ${ }^{23}$ utilizaram dispositivo mais robusto e com capacidade para fornecer carga de até $1675 \mathrm{~N}$ em todo arco do movimento simulando a inércia associada à força gravitacional, sendo que no estudo de Smith et al (2012) ${ }^{23}$ foi associada manutenção do estado nutricional (evitando-se a comum anorexia dos tripulantes de vôos espaciais), e, pode-se observar melhores resultados na manutenção da DMO em várias regiões ósseas. No estudo de SINGH e colaboradores, ${ }^{24}$ os indivíduos que executaram o programa de exercícios resistidos no isocinético e com pesos livres obtiveram melhora da DMO em todos os sítios ósseos, inclusive na coluna, porém sem diferença estatisticamente significativa em relação ao grupo controle. Foi evidenciado nesse protocolo de treinamento aumento da DMO em 2,2\% na coluna, apesar da curta duração - 9 meses - do treinamento e com frequência de apenas duas vezes por semana. Levantou-se a hipótese de que os tipos de exercícios executados e o número de séries e repetições ainda não seriam os mais efetivos para o ganho de DMO.

Aspecto importante apresentado em ambos os estudos, como também, nos protocolos de exercício de impacto, é a progressão tanto da intensidade da carga aplicada quanto da frequência por semana, parecendo aumentar, de forma diretamente proporcional, a DMO.

\section{Conclusão}

Pode-se constatar, após revisão sistemática dos artigos selecionados, que há maior eficiência do protocolo de exercício com atividades de alto impacto e curto período de execução, com mais sessões durante a semana, para aumento da DMO do fêmur proximal (colo femoral e região intertrocantérica), sendo esses locais de grande incidência de fraturas por osteoporose. Em menor frequência, foi observado aumento da DMO na coluna lombar quando exercícios resistidos são incluídos ou cargas de impacto com maior intensidade são aplicadas. Portanto, para ganho mais generalizado de densidade mineral óssea parece que o ideal é associar o exercício de impacto aos exercícios resistidos, inclusive para maior proteção do tecido ósseo da coluna contra fraturas em idades mais avançadas. Como também, é importante que os exercícios sejam frequentes, não sendo necessários serem prolongados, mas sim variados, com direções diversificadas para maior exigência muscular e maior exposição de áreas de tecido ósseo às sobrecargas decorrentes de tensão muscular ou das forças de reação do solo.

A maioria dos estudos usou protocolo experimental em relativo curto período de tempo (6 meses), demonstrando que programas simples, de fácil execução e que requerem pouco tempo de aplicação, sem necessidade de aparelhos específicos, são suficientes para promover remodelação óssea em sítios específicos de descarga de peso com aumento da DMO. Apesar dos benefícios sugeridos por muitos estudos a respeito dos efeitos da atividade física sobre o metabolismo ósseo, muitas mulheres ainda não aderiram a estilo de vida mais ativo por falta de tempo, motivação e conhecimento. Uma das dificuldades encontradas nesta revisão foi a grande variedade de metodologias utilizada nos estudos e a pouca aderência das mulheres aos protocolos de exercícios. Cabe, portanto, aos profissionais da área contínuo esclarecimento e incentivo da população.

\section{ABSTRACT}

Study Design: Sistematic review of literature.

Aims: Physical activity is an important stimulus to increase bone mineral density (BMD). Whereby the resistance of the bone is associated with BMD, microstructure and material properties, improvements in bone mass are important to prevent osteoporosis in old ages. Despite many studies on the subject, there is no consensus on what is the best type of exercise, intensity and frequency for improvements in osteogenesis and promotion of BMD gain. This study aimed to investigate the types of physical activities that results in better osteogenic effects on increasing BMD in healthy premenopausal women. 


\begin{abstract}
Methodology: We searched scientific articles in Pubmed and Science Direct databases published in the last ten years. We selected controlled studies which used high-impact activities or resistance training. We used systematic analysis of the selected articles.

Results: Fifteen articles were included in this review, which were separated into two groups according to the type of exercise. We performed a comparative analysis of the methods used and the results achieved. There were more efficient protocols using high impact exercise even if it was a short-term program resulting in increased BMD in the proximal femur (femoral neck and intertrochanteric region).

Conclusion: There were fewer studies observing increases in BMD at the lumbar spine when resistance exercise with load or high impact loads were used. Most experimental protocols performed in relatively short-term programs (6 months) demonstrated that exercise programs that were simple, easy to perform, and not using special devices are sufficient to promote bone remodeling at specific sites resulting in increased BMD.
\end{abstract}

Key-words: Exercise. Bone Mineral Density. Women.

\section{Referênclas Blbllográficas}

1. Vainionpaa A, Korpelainen R, Vaananen HK, Haapalahti J, Jamsa $\mathrm{T}$, Leppaluoto J. Effect of impact exercise on bone metabolism. Osteoporosis Int. 2009;20:1725-33.

2. Vainionpaa A, Korpelainen R, Leppaluoto J, Jamsa T. Effects of high-impact exercise on bone mineral density: a randomized controlled trial in premenopausal women. Osteoporosis Int. 2005;16:191-7.

3. Bailey CA, Wavell KB. Exercise for optimising peak bone mass in women. Proc Nutr Soc. 2008;67:9-18.

4. Niu K, Ahola R, Guo H, Korpelainen R, Uchimaru J, Vainionpaa, et al. Effect of office-based brief high-impact exercise on bone mineral density in healthy premenopausal women: the Sendai Bone Health Concept Study. J Bone Miner Metab. 2010;28:568-77.

5. Wang M, Salem GJ. The relations among upper-extremity loading characteristics and bone mineral density changes in young women. Bone. 2004;34:1053-63.

6. Rantalainen T, Nikander R, Daly RM, Heinonen A, Sievanen T. Exercise loading and cortical bone distribution at the tibial shaft. Bone. 2011;48:786-91.

7. Balasch J. Sex steroids and bone: current perspectives. Hum Reprod Update. 2003; 9: 207-22.

8. Smith SM, Zwart SR, Heer M, Lee SMC, Baecker N, Meuche S, et al. WISE-2005: Supine treadmill exercise within lower body negative pressure and flywheel resistive exercise as a countermeasure to bed rest-induced bone loss in women during 60-day simulated microgravity. Bone. 2008; 42:572-81.

9. Papachroni KK, Karatzas DN, Papavassiliou K A, Basdra EK, Papavassiliou AG. Mechanotransduction in osteoblast regulation and bone disease. Trends Mol Med. 2009;15:208-16

10. Sugiyama T, Yamaguchi A, Kawai S. Effects of skeletal loading on bone mass and compensation mechanism in bone: a new insight into the "mechanostat" theory. J Bone Miner Metab. 2002;20:196-200.

11. Babatunde OO, Forsyth JJ, Gidlow CJ. A meta-analysis of brief high-impact exercises for enhancing bone health in premenopausal women. Osteoporosis Int. 2012;23:109-19.

12. Vainionpää $A$, Korpelainen $R$, Vihriälä $E$, Rinta-paavola $A$, Leppäluoto J, Jämsä $T$. Intensity of exercise is associated with bone density change in premenopausal women Osteoporosis Int. 2006;17:455-63.
13. Bailey CA, Wavell KB. Optimum frequency of exercise for bone health: Randomised controlled trial of a high-impact unilateral intervention. Bone. 2010; 46:1043-49.

14. Ahola R, Korpelainen R, Vainionpaa A, Leppaluoto J, Jamsa T. Time-course of exercise and its association with 12-month bone changes. BMC Musculoskelet Disord. 2009; 10:138.

15. Kemper HCG, Bakker I, Twisk JWR, Mechelen WV. Validation of a Physical Activity Questionnaire to Measure the Effect of Mechanical Strain on Bone Mass. Bone. 2002;30:799-804.

16. Stager M, Harvey R, Secic M, Camlin-Shingler K, Cromer B. Self-Reported Physical Activity and Bone Mineral Density in Urban Adolescent Girls. J Pediatr Adolesc Gynecol. 2006;19:17-22.

17. Stone KMW, Snow CM. Site-specific response of bone to exercise in premenopausal women. Bone. 2006; 39:1203-9.

18. Heikkinen R, Vihriala E, Vainionpaa A, Korpelainen R, Jamsa T. Acceleration slope of exercise-induced impacts is a determinant of changes in bone density. J Biomech. 2007; 40 : 2967-74.

19. James MM, Carroll S. Effects of different impact exercise modalities on bone mineral density in premenopausal women: a meta-analysis. J Bone Miner Metab. 2010; 28:251-67.

20. Adami S, Gatti D, Viapiana O, Fiore CE, Nuti R, Luisetto G, et al. Physical Activity and Bone Turnover Markers: A CrossSectional and a Longitudinal Study. Calcif Tissue Int. 2008; 83: 388-92.

21. Zwart SR, Hargens AR, Lee SMC, Macias BR, Watenpaugh $\mathrm{DE}$, Tse $\mathrm{K}$, et al. Lower body negative pressure treadmill exercise as a countermeasure for bed rest-induced bone loss in female identical twins. Bone. 2007; 40: 529-37.

22. Loehr JA, Lee SM, English KI, Sibonga J, Smith SM, Spiering $\mathrm{BA}$, et al. Musculoskeletal adaptations to training with the advanced resistive exercise device. Med Sci Sports Exerc. 2011; 43: 146-56.

23. Smith SM, Heer MA, Shackelford LC, Sibonga JD, PloutzSnyder L, Zwart SR. Benefits for Bone From Resistance Exercise and Nutrition in Long-Duration Spaceflight: Evidence From Biochemistry and Densitometry. J Bone Miner Res. 2012; 27: 1896-906.

24. Singh JA, Schmitz KH, Petit MA. Effect of resistance exercise on bone mineral density in premenopausal women. Joint Bone Spine. 2009;76: 273-80. 